\title{
Poisson Cohomology of Plane Quadratic Poisson Structures
}

By

\author{
Nobutada NAKANISHI*
}

\section{§1. Introduction}

As is well-known, Poisson cohomology is of special importance in the theory of Poisson geometry. But unfortunately, the computation is very complicated because of the lack of a powerful method.

Let $(M, \pi)$ be a Poisson manifold, where $M$ is a $C^{\infty}$-manifold and $\pi$ denotes a Poisson structure on $M$. If the rank of $\pi$ is everywhere constant on $M,(M, \pi)$ is said to be regular. The computation of Poisson cohomology of regular Poisson manifolds was first studied by A. Lichnerowicz [6]. Some other references are [5], [12], [14].

If $(M, \pi)$ is not regular, certain difficulties will arise in computations of Poisson cohomology. Typical examples of such manifolds are linear Poisson manifolds. They are, by definition, the dual spaces of finite dimensional Lie algebras. Their Poisson structures are naturally induced from their Lie algebra structures. There are also some results on the computations of their Poisson cohomology (see e.g., [3], [8], [9], [10], [11]).

In the present article, we shall treat quadratic Poisson structures $\pi$ on the plane $R^{2}$, and compute their Poisson cohomology. Note that each Poisson manifold $\left(R^{2}, \pi\right)$ is irregular, except for the trivial one, $\left(R^{2}, 0\right)$. In considering this problem, the author was motivated by I. Vaisman ([13], p.67).

\section{Acknowledgement}

The author would like to thank Professors Izu Vaisman, Alan Weinstein,

Communicated by T. Miwa, January 22, 1996.

1991 Mathematics Subject Classification(s): 58F05

* Department of Mathematics, Gifu Keizai University, 5-50 Kitagata-cho Ogaki-city Gifu, 503, Japan 
Viktor Ginzburg and Jean Paul Dufour for their valuable e-mail communications on this subject. He is also grateful to Professors T. Morimoto, E. Kaneda and C. Tsukamoto for helpful and stimulating discussions.

\section{§2. Poisson Manifolds and Poisson Cohomology}

Let $(M, \pi)$ be a Poisson manifold. Then the Poisson tensor $\pi$ is written in local coordinates $\left(x_{1}, x_{2}, \cdots, x_{n}\right)$ as

$$
\pi=\frac{1}{2} \sum_{1 \leq i, j \leq n} \pi_{i j} \frac{\partial}{\partial x_{i}} \wedge \frac{\partial}{\partial x_{j}},
$$

with $\pi_{i j}=-\pi_{j i}$, such that $\pi$ satisfies the equation

$$
\sum_{1 \leq l \leq n}\left(\pi_{i l} \frac{\partial \pi_{j k}}{\partial x_{l}}+\pi_{j l} \frac{\partial \pi_{k i}}{\partial x_{l}}+\pi_{k l} \frac{\partial \pi_{i j}}{\partial x_{l}}\right)=0
$$

for $1 \leq i, j, k \leq n$. Since the Poisson bracket is defined by $\{f, g\}=\langle\pi \mid d f \wedge d g\rangle$, the coefficients $\pi_{i j}$ of $\pi$ are obtained by the Poisson bracket, $\pi_{i j}=\left\{x_{i}, x_{j}\right\}$. If each $\pi_{i j}$ is a homogeneous linear polynomial, then the Poisson structure $\pi$ is said to be linear. Similarly if each $\pi_{i j}$ is a homogeneous quadratic polynomial, then it is said to be quadratic.

Let $\chi^{i}(M)$ denote the space of $i$-vectors (i.e. skew symmetric contravariant tensor fields of type $(i, 0))$, and let $L(M)=\left(\oplus_{i=0}^{n} \chi^{i}(M), \wedge\right)$ be the contravariant Grassmann algebra of $M$, where $n$ is the dimension of $M$. In particular, $\chi^{0}(M)=C^{\infty}(M)$ and $\chi^{1}(M)$ is the space of all vector fields on $M$ denoted by $\chi(M)$.

From now on, let us denote the Schouten bracket by [,]. The Schouten bracket is a homogeneous bi-derivation of degree -1 defined on $L(M)$ :

$$
\chi^{i}(M) \times \chi^{j}(M) \ni(T, U) \mapsto[T, U] \in \chi^{i+j-1}(M)
$$

and is determined by the following six properties:

1. $[f, h]=0, \forall f, h \in \chi^{0}(M)$,

2. $[X, f]=X f, \quad \forall X \in \chi^{1}(M), \quad f \in \chi^{0}(M)$,

3. $[X, Y]=[X, Y]_{\text {Liebracket }}, \forall X, Y \in \chi^{1}(M)$,

4. $[T, U \wedge W]=[T, U] \wedge W+(-1)^{(t-1) u} U \wedge[T, W], \quad \forall T \in \chi^{t}(M)$, $U \in \chi^{u}(M), \quad W \in \chi^{w}(M)$,

5. $[T, U]=(-1)^{(t-1)(u-1)+1}[U, T], \quad \forall T \in \chi^{t}(M), \quad U \in \chi^{u}(M)$,

6. $(-1)^{(t-1)(w-1)}[[T, U], W]+(-1)^{(u-1)(t-1)}[[U, W], T]$ 


$$
+(-1)^{(w-1)(u-1)}[[W, T], U]=0, \quad \forall T \in \chi^{t}(M), \quad U \in \chi^{u}(M), \quad W \in \chi^{w}(M) .
$$

It is easily seen that a 2-tensor $\pi \in \chi^{2}(M)$ becomes a Poisson tensor if and only if $\pi$ satisfies $[\pi, \pi]=0$. The space of infinitesimal automorphisms of the Poisson structure $\pi$, which we denote by $Z_{\pi}^{1}(M)$, is the set of vector fields $X$ satisfying $[X, \pi]=0$. We denote by $B_{\pi}^{1}(M)$ the space of Hamiltonian vector fields $X_{f},\left(f \in C^{\infty}(M)\right)$. Recall that a Hamiltonian vector field is defined by $X_{f}(g)=\{f, g\}$ for all $g \in C^{\infty}(M)$. If one uses the Schouten bracket, $X_{f}$ is also defined by $X_{f}=[\pi, f]$. With respect to the Schouten bracket, $L(M)$ becomes a Lie superalgebra. We define the linear mapping $D: L(M) \rightarrow L(M)$ by $X \mapsto[\pi, X]$. Since the Poisson structure $\pi$ satisfies $[\pi, \pi]=0, D$ satisfies $D^{2}=0$ and becomes a coboundary operator. $D$ maps $\chi^{i}(M)$ into $\chi^{i+1}(M)$. The cohomology with respect to this coboundary operator $D$ is called Poisson cohomology and is denoted by $H_{\pi}^{*}(M)$. The k-th Poisson cohomology space of $(M, \pi)$ is given by

$$
H_{\pi}^{k}(M)=\frac{\operatorname{ker}\left(D: \chi^{k}(M) \rightarrow \chi^{k+1}(M)\right)}{\operatorname{im}\left(D: \chi^{k-1}(M) \rightarrow \chi^{k}(M)\right)}
$$

Then the following facts come clear in a straightforward way:

a) $H_{\pi}^{0}(M)$ is the center of the Poisson algebra $C^{\infty}(M)$. (This space is also called the space of Casimir functions.)

b) $H_{\pi}^{1}(M) \cong Z_{\pi}^{1}(M) / B_{\pi}^{1}(M)$.

\section{§3. Quadratic Poisson Structures on $R^{2}$}

In this section, we classify all quadratic Poisson structures on $R^{2}$. See [1], [2], [7] for the classification of quadratic Poisson structures under more general situations. Using the theorem of Z-J. Liu and Ping $\mathrm{Xu}$ [7], we can see that the only "exact" quadratic Poisson structure on $R^{2}$ is zero. Hence it is quite easy to classify quadratic Poisson structures on $R^{2}$.

Let $x, y$ be the standard coordinates on $R^{2}$. Then any quadratic Poisson bracket on $R^{2}$ is given by $\{x, y\}=a x^{2}+b x y+c y^{2}$, where $a, b$ and $c$ are arbitrary constants. Let $K$ be the matrix in $\mathfrak{s l}(2, R)$;

$$
K=\left(\begin{array}{cc}
b / 2 & c \\
-a & -b / 2
\end{array}\right)
$$

and $I$ be the identity matrix. Then it is easy to see that $\Lambda=K \wedge I$ is the 
triangular $r$-matrix. Thus, following $\mathrm{Z}$-J. Liu and $\mathrm{Ping} \mathrm{Xu}$, it induces the quadratic Poisson structure $\pi_{\Lambda}$ on $R^{2}$. To state it specifically, $\pi_{\Lambda}$ is given by

$$
\pi_{\Lambda}=\left(a x^{2}+b x y+c y^{2}\right) \frac{\partial}{\partial x} \wedge \frac{\partial}{\partial y} .
$$

This means that any quadratic Poisson structure on $R^{2}$ is obtained in the same way. The following result is due to $\mathrm{Z}$-J. Liu and Ping $\mathrm{Xu}$.

Proposition 3.1 [7]. Let $\Lambda=K \wedge I$ and $\Lambda^{\prime}=K^{\prime} \wedge I$ be two triangular r-matrices. The quadratic Poisson structures $\pi_{\Lambda}$ and $\pi_{\Lambda^{\prime}}$ on $R^{2}$ are Poisson diffeomorphic if and only if $K^{\prime}=T^{-1} K T$ for a certain linear isomorphism $T$.

This proposition indicates that in order to classify all quadratic Poisson structures on $R^{2}$, we only need to classify $\mathfrak{s l}(2, R)$ by the Jordan forms. By this procedure, we obtain the classification of all quadratic Poisson structures on $R^{2}$.

Proposition 3.2. The following is a complete list of all quadratic Poisson structures $\pi$ on $R^{2}$ up to Poisson diffeomorphisms. (The subscript $\Lambda$ is omitted.)

(1) $K=\left(\begin{array}{ll}0 & 0 \\ 0 & 0\end{array}\right)$, then $\pi=0$.

(2) $K=\left(\begin{array}{cc}0 & \delta \\ -\delta & 0\end{array}\right), \quad \delta \neq 0$, then $\pi=\delta\left(x^{2}+y^{2}\right) \frac{\partial}{\partial x} \wedge \frac{\partial}{\partial y}$.

(3) $K=\left(\begin{array}{cc}\epsilon / 2 & 0 \\ 0 & -\epsilon / 2\end{array}\right), \quad \epsilon \neq 0$, then $\pi=\epsilon x y \frac{\partial}{\partial x} \wedge \frac{\partial}{\partial y}$.

(4) $K=\left(\begin{array}{ll}0 & 1 \\ 0 & 0\end{array}\right)$, then $\pi=y^{2} \frac{\partial}{\partial x} \wedge \frac{\partial}{\partial y}$.

In the above proposition, the "standard elliptic" Poisson structure $\left(x^{2}+y^{2}\right) \frac{\partial}{\partial x} \wedge \frac{\partial}{\partial y}$ is not isomorphic to $\delta\left(x^{2}+y^{2}\right) \frac{\partial}{\partial x} \wedge \frac{\partial}{\partial y}$ if $\delta \neq \pm 1$. Similarly the "standard hyperbolic" Poisson structure $x y \frac{\partial}{\partial x} \wedge \frac{\partial}{\partial y}$ is not isomorphic to $\epsilon x y \frac{\partial}{\partial x} \wedge \frac{\partial}{\partial y}$ if $\epsilon \neq \pm 1$. However for case (4), the Poisson structure $y^{2} \frac{\partial}{\partial x} \wedge \frac{\partial}{\partial y}$ is isomorphic to $\tau y^{2} \frac{\partial}{\partial x} \wedge \frac{\partial}{\partial y}$ for all $\tau \neq 0$. (These facts were pointed out to the author by $A$. Weinstein and J.P. Dufour.) Nevertheless, as far as Poisson cohomology is concerned, we only need to consider the standard Poisson structures (i.e. 
$\delta=\epsilon=1)$

\section{§4. Computations of $H_{\pi}^{1}\left(R^{2}\right)$}

4.1. Case (1) $\left(R^{2}, \pi=0\right)$.

The cohomology spaces $H_{\pi}^{*}\left(R^{2}\right)$ are easily obtained. In fact, we immediately have $H_{\pi}^{*}\left(R^{2}\right)=\chi^{*}\left(R^{2}\right)$. For other cases, the following results are useful for computations of Poisson cohomology:

Proposition 4.1 [13]. If a Poisson manifold $(M, \pi)$ is a symplectic manifold, that is, if $\pi$ is of full rank, then $H_{\pi}^{*}(M) \cong H_{d R}^{*}(M)$, where $H_{d R}^{*}(M)$ stands for the usual de Rham cohomology.

Proposition 4.2 [13]. If $\left(M_{1}, \pi_{1}\right)$ and $\left(M_{2}, \pi_{2}\right)$ are Poisson manifolds and $\phi: M_{1} \rightarrow M_{2}$ is a Poisson mapping which is a local diffeomorphism, then one obtains the following induced homomorphism: $\phi^{*}: H_{\pi_{2}}^{k}\left(M_{2}\right) \rightarrow H_{\pi_{1}}^{k}\left(M_{1}\right)$.

\subsection{Case (2) $\left(R^{2}, \pi=\delta\left(x^{2}+y^{2}\right) \frac{\partial}{\partial x} \wedge \frac{\partial}{\partial y}\right)$.}

When computing cohomology groups, we can assume $\delta=1$. For this case, V. Ginzburg also computed cohomology spaces $H_{\pi}^{*}\left(R^{2}\right)[4]$. It is easy to see that Casimir functions are only constants. Hence we have $H_{\pi}^{0}\left(R^{2}\right) \cong R$. We will proceed to compute $H_{\pi}^{1}\left(R^{2}\right)$. Since the canonical inclusion mapping $l: R^{2} \backslash(0) \rightarrow R^{2}$ is a Poisson map, by Proposition 4.2, it induces a homomorphism $\imath^{*}: H_{\pi}^{*}\left(R^{2}\right) \rightarrow H_{\pi}^{*}\left(R^{2} \backslash(0)\right)$. Note that $\left(R^{2} \backslash(0), \pi\right)$ is a symplectic manifold. Hence by Proposition 4.1 we get: $H_{\pi}^{1}\left(R^{2} \backslash(0)\right) \cong H_{d R}^{1}\left(R^{2} \backslash(0)\right) \cong R$. Consider the vector field $X=x \frac{\partial}{\partial x}+y \frac{\partial}{\partial y}$. Then $[X, \pi]=0$ and it is easy to confirm that $[X] \neq 0$ in $H_{\pi}^{1}\left(R^{2}\right)$. Moreover, $l^{*}[X] \neq 0$ even in $H_{\pi}^{1}\left(R^{2} \backslash(0)\right)$, and it generates $H_{\pi}^{1}\left(R^{2} \backslash(0)\right)$. It follows that the mapping $\imath^{*}: H_{\pi}^{1}\left(R^{2}\right) \rightarrow H_{\pi}^{1}\left(R^{2} \backslash(0)\right)$ is surjective. Let $\mathscr{F}=C^{\infty}\left(R^{2}\right)$, and define a space $\mathscr{G}$ by

$$
\mathscr{G}=\left\{f \in C^{\infty}\left(R^{2}-(0)\right) \mid\left(x^{2}+y^{2}\right) \frac{\partial f}{\partial x}, \quad\left(x^{2}+y^{2}\right) \frac{\partial f}{\partial y} \in \mathscr{F}\right\} .
$$

Here $\left(x^{2}+y^{2}\right) \frac{\partial f}{\partial x} \in \mathscr{F}$ means that $\lim _{(x, y) \rightarrow(0,0)}\left(x^{2}+y^{2}\right) \frac{\partial f}{\partial x}$ exists and the new function (which we also denote by $\left.\left(x^{2}+y^{2}\right) \frac{\partial f}{\partial x}\right)$ defined at the origin is an element of $\mathscr{F}$. The meaning of $\left(x^{2}+y^{2}\right) \frac{\partial f}{\partial y} \in \mathscr{F}$ is the same as $\left(x^{2}+y^{2}\right) \frac{\partial f}{\partial x} \in \mathscr{F}$.

Then $\mathscr{G}$ contains $\mathscr{F}$ as its subspace. We define a linear mapping $T: \mathscr{G} \rightarrow H_{\pi}^{1}\left(R^{2}\right)$ by $T(f)=\left[X_{f}\right]$. Then it is clear that $T(\mathscr{G})=\operatorname{ker} \imath^{*}$. Let $f=\frac{1}{2} \log \left(x^{2}+y^{2}\right)$. Then $f$ is an element of $\mathscr{G}$, and $T(f)=\left[X_{f}\right]=\left[y \frac{\partial}{\partial x}-x_{\frac{\partial}{\partial y}}\right]$ is a 
non-zero element of $H_{\pi}^{1}\left(R^{2}\right)$. But $l^{*}\left[X_{f}\right]=0$ in $H_{\pi}^{1}\left(R^{2} \backslash(0)\right)$. Hence $\operatorname{ker} l^{*} \neq 0$. We denote by $\mathscr{F}_{c}$ the space generated by $\mathscr{F}$ and constant functions on $R^{2} \backslash(0)$. The following lemma is quite easy.

Lemma 4.3. (a) $H_{\pi}^{1}\left(R^{2}\right) / \operatorname{ker} \imath^{*} \cong H_{\pi}^{1}\left(R^{2} \backslash(0)\right) \cong R$.

(b) $\mathscr{G} / \mathscr{F}_{c} \cong \operatorname{ker} \imath^{*}$.

Next we precisely determine the space $\mathscr{G} / \mathscr{F}_{c}$. A function $f \in C^{\infty}\left(R^{2} \backslash(0)\right)$ belongs to $\mathscr{G}$ if and only if it satisfies $\left(x^{2}+y^{2}\right) \frac{\partial f}{\partial y}=-a$, and $\left(x^{2}+y^{2}\right) \frac{\partial f}{\partial x}=b$ for some functions $a, b \in \mathscr{F}$. By the integrability condition of $f$ on $R^{2} \backslash(0)$, it holds that $\left(x^{2}+y^{2}\right)\left(\frac{\partial a}{\partial x}+\frac{\partial b}{\partial y}\right)=2(x a+y b)$. Note that this equation is the selfsame condition for the vector field $X=a \frac{\partial}{\partial x}+b \frac{\partial}{\partial y}$ to be a 1-cocycle. Thus for any function $f \in \mathscr{G}$, there exist $a, b \in \mathscr{F}$ such that the following conditions hold:

$$
\left\{\begin{array}{l}
\left(x^{2}+y^{2}\right) \frac{\partial f}{\partial y}=-a, \\
\left(x^{2}+y^{2}\right) \frac{\partial f}{\partial x}=b, \\
\left(x^{2}+y^{2}\right)\left(\frac{\partial a}{\partial x}+\frac{\partial b}{\partial y}\right)=2(x a+y b) .
\end{array}\right.
$$

Lemma 4.4. Let $P$ and $Q$ be two polynomials of degree $n,(n \geq 2)$. If $P$ and $Q$ satisfy

$$
\left(x^{2}+y^{2}\right)\left(\frac{\partial P}{\partial x}+\frac{\partial Q}{\partial y}\right)=2(x P+y Q)
$$

then there exist two polynomials $P_{1}$ and $Q_{1}$ of degree $n-2$ such that $P$ and $Q$ are written in the following form:

$$
\left\{\begin{array}{l}
P=a_{1} x+b_{1} y+\left(x^{2}+y^{2}\right) P_{1}, \\
Q=-b_{1} x+a_{1} y+\left(x^{2}+y^{2}\right) Q_{1} \\
\frac{\partial P_{1}}{\partial x}=-\frac{\partial Q_{1}}{\partial y}
\end{array}\right.
$$

where $a_{1}$ and $b_{1}$ are constants. 
Proof. By (4.2), it is easy to see that it suffices to prove the case of homogeneous polynomials. If $\operatorname{deg} P=\operatorname{deg} Q=1$, then it is clear that $P=a_{1} x+b_{1} y$ and $Q=-b_{1} x+a_{1} y$. Next let $P=\Sigma_{k+l=n} p_{k, l} x^{k} y^{l}$ and $Q=\Sigma_{k+l=n}$ $q_{k, l} x^{k} y^{l}$ be homogeneous polynomials of degree $n,(n \geq 2)$. It is convenient to consider $n$ under each of cases of modulo 4. Then direct computations show that

$$
\begin{aligned}
& \left\{\begin{array}{l}
p_{4 m, 0}-p_{4 m-2,2}+\cdots+p_{0,4 m}=0, \\
p_{4 m-1,1}-p_{4 m-3,3}+\cdots-p_{1,4 m-1}=0, \quad \text { if } n \equiv 0,
\end{array}\right. \\
& \left\{\begin{array}{l}
p_{4 m-1,0}-p_{4 m-3,2}+\cdots-p_{1,4 m-2}=0, \\
p_{4 m-2,1}-p_{4 m-4,3}+\cdots-p_{0,4 m-1}=0, \quad \text { if } n \equiv 3,
\end{array}\right. \\
& \left\{\begin{array}{l}
p_{4 m-2,0}-p_{4 m-4,2}+\cdots-p_{0,4 m-2}=0, \\
p_{4 m-3,1}-p_{4 m-5,3}+\cdots+p_{1,4 m-3}=0, \quad \text { if } n \equiv 2,
\end{array}\right. \\
& \left\{\begin{array}{l}
p_{4 m-3,0}-p_{4 m-5,2}+\cdots+p_{1,4 m-4}=0, \\
p_{4 m-4,1}-p_{4 m-6,3}+\cdots+p_{0,4 m-3}=0, \quad \text { if } n \equiv 1 .
\end{array}\right.
\end{aligned}
$$

In all cases above, as can be easily seen, it holds that $P(x, \sqrt{-1} x)=0$. The same results are valid for $q_{k, l}$, and thus it also holds that $Q(x, \sqrt{-1} x)=0$. This means that both $P$ and $Q$ have the factor $x^{2}+y^{2}$.

Using this lemma, we offer the following proof:

Proposition 4.5. $\mathscr{G} / \mathscr{F}_{c}$ is isomorphic to $R$.

Proof. For any $f \in \mathscr{G}, f$ satisfies (4.1). For arbitrary $n \geq 2$, let us consider the Taylor expansions of order $n+1$ at the origin of the functions $a$ and $b$. We write these Taylor expansions as $a=a_{n}+R_{1, n}$ and $b=b_{n}+R_{2, n}$, where $a_{n}, b_{n}$ are polynomials of degree $n$ and $R_{1, n}, R_{2, n}$ are remainder terms. Then we know that both $a_{n}$ and $b_{n}$ satisfy the condition of Lemma 4.4. From now on, we denote by $[k(x, y)]_{(0,0)}$ the formal Taylor expansion of any $k(x, y) \in \mathscr{F}$ at the origin. Since $n$ is arbitrary, the formal Taylor expansions at the origin of $a(x, y)-\left(a_{1} x+b_{1} y\right)$ and $b(x, y)-\left(-b_{1} x+a_{1} y\right)$ can be written as

$$
\left\{\begin{array}{l}
{\left[a(x, y)-\left(a_{1} x+b_{1} y\right)\right]_{(0,0)}=\left(x^{2}+y^{2}\right) \cdot A(x, y),} \\
{\left[b(x, y)-\left(-b_{1} x+a_{1} y\right)\right]_{(0,0)}=\left(x^{2}+y^{2}\right) \cdot B(x, y),}
\end{array}\right.
$$

where $A(x, y)$ and $B(x, y)$ are suitable formal power series. By the well-known 
theorem of $\mathrm{E}$. Borel, there exist $C^{\infty}$-functuions $\alpha(x, y)$ and $\beta(x, y)$ such that $[\alpha(x, y)]_{(0,0)}=A(x, y)$ and $[\beta(x, y)]_{(0,0)}=B(x, y)$.

Recall that a $C^{\infty}$-function is called flat at the origin if its formal Taylor expansion at the origin vanishes. Then the $C^{\infty}$-function $a(x, y)-\left(a_{1} x+b_{1} y\right)$ $-\left(x^{2}+y^{2}\right) \cdot \alpha(x, y)$ is flat at the origin, and is denoted by

$$
a(x, y)-\left(a_{1} x+b_{1} y\right)-\left(x^{2}+y^{2}\right) \cdot \alpha(x, y)=\alpha_{1}(x, y) .
$$

Similarly, we have

$$
b(x, y)-\left(-b_{1} x+a_{1} y\right)-\left(x^{2}+y^{2}\right) \cdot \beta(x, y)=\beta_{1}(x, y) .
$$

Since $\alpha_{1}(x, y)$ and $\beta_{1}(x, y)$ are flat at the origin, we can express them in another way: $\alpha_{1}(x, y)=\left(x^{2}+y^{2}\right) \cdot \frac{\alpha_{1}(x, y)}{x^{2}+y^{2}}$, and $\beta_{1}(x, y)=\left(x^{2}+y^{2}\right) \cdot \frac{\beta_{1}(x, y)}{x^{2}+y^{2}}$. Note that both $\frac{\alpha_{1}(x, y)}{x^{2}+y^{2}}$ and $\frac{\beta_{1}(x, y)}{x^{2}+y^{2}}$ are still $C^{\infty}$-functions.

Let $g(x, y)=\alpha(x, y)+\frac{\alpha_{1}(x, y)}{x^{2}+y^{2}}$ and $h(x, y)=\beta(x, y)+\frac{\beta_{1}(x, y)}{x^{2}+y^{2}}$. Then $a$ and $b$ can be written as

$$
\left\{\begin{array}{l}
a=a_{1} x+b_{1} y+\left(x^{2}+y^{2}\right) \cdot g(x, y), \\
b=-b_{1} x+a_{1} y+\left(x^{2}+y^{2}\right) \cdot h(x, y), \\
\frac{\partial g}{\partial x}=-\frac{\partial h}{\partial y}
\end{array}\right.
$$

where $a_{1}$ and $b_{1}$ are constants. Let $\gamma=d f=\frac{\partial f}{\partial x} d x+\frac{\partial f}{\partial y} d y$. According to the last equation in (4.5), there exists a function $e(x, y) \in \mathscr{F}$ such that $h d x-g d y=d e$. Thus we have

$$
\begin{aligned}
\gamma & =a_{1} \frac{y d x-x d y}{x^{2}+y^{2}}-b_{1} \frac{x d x+y d y}{x^{2}+y^{2}}+h d x-g d y \\
& =a_{1} \frac{y d x-x d y}{x^{2}+y^{2}}-d\left\{\frac{b_{1}}{2} \log \left(x^{2}+y^{2}\right)\right\}+d e .
\end{aligned}
$$

Since $[\gamma]=0$ in $H_{d R}^{1}\left(R^{2} \backslash(0)\right)$ and the generator of $H_{d R}^{1}\left(R^{2} \backslash(0)\right)$ is $\left[\frac{y d x-x d y}{x^{2}+y^{2}}\right]$, it holds that $a_{1}=0$ in (4.6). Thus from (4.6), it follows that $d\left\{f+\frac{b_{1}}{2} \log \left(x^{2}+y^{2}\right)-e\right\}$ $=0$, and we get $f \equiv-\frac{b_{1}}{2} \log \left(x^{2}+y^{2}\right),\left(\bmod \mathscr{F}_{c}\right)$. This completes the proof.

Combining Lemma 4.3 and Proposition 4.5, we get the following theorem:

Theorem 4.6. $H_{\pi}^{1}\left(R^{2}\right) \cong R \oplus R$. 


\subsection{Case (3) $\left(R^{2}, \pi=\epsilon x y \frac{\partial}{\partial x} \wedge \frac{\partial}{\partial y}\right)$.}

The same remark holds as in case (2). Namely, we can let $\epsilon=1$ in computing Poisson cohomology groups. The space of Casimir functions coincides with $R$. Hence $H_{\pi}^{0}\left(R^{2}\right) \cong R$. Let $N=\{(x-$ axis $) \cup(y-$ axis $)\}$. To compute $H_{\pi}^{1}\left(R^{2}\right)$, let us also consider the canonical inclusion $\imath: R^{2} \backslash N \rightarrow R^{2}$. By Proposition 4.2, we have the induced homomorphism $\imath^{*}: H_{\pi}^{*}\left(R^{2}\right) \rightarrow H_{\pi}^{*}\left(R^{2} \backslash N\right)$. Since $\left(R^{2} \backslash N, \pi\right)$ is a symplectic manifold, it follows that $H_{\pi}^{1}\left(R^{2} \backslash N\right) \cong H_{d R}^{1}\left(R^{2} \backslash N\right)$ $=0$. Thus the mapping $\imath^{*}: H_{\pi}^{1}\left(R^{2}\right) \rightarrow H_{\pi}^{1}\left(R^{2} \backslash N\right)=0$ is clearly surjective. Let $X=a \frac{\partial}{\partial x}+b \frac{\partial}{\partial y}$ be an element of $Z_{\pi}^{1}\left(R^{2}\right)$. Then $a$ and $b$ satisfy

$$
b x+a y=x y\left(\frac{\partial a}{\partial x}+\frac{\partial b}{\partial y}\right) .
$$

On the other hand, a Hamiltonian vector field $\boldsymbol{X}_{\boldsymbol{f}}$ is given by

$$
\boldsymbol{X}_{f}=x y\left(-\frac{\partial f}{\partial y} \frac{\partial}{\partial x}+\frac{\partial f}{\partial x} \frac{\partial}{\partial y}\right)
$$

Let us define a subspace $\mathscr{H}$ by

$$
\mathscr{H}=\left\{f \in C^{\infty}\left(R^{2} \backslash \boldsymbol{N}\right) \mid x y \frac{\partial f}{\partial x}, x y \frac{\partial f}{\partial y} \in \mathscr{F}\right\} .
$$

Then $\mathscr{F}$ is a subspace of $\mathscr{H}$. It is clear that the mapping $U: f \in \mathscr{H} \rightarrow\left[\boldsymbol{X}_{f}\right]$ $\in H_{\pi}^{1}\left(R^{2}\right)$ is well-defined. Now we will prove that this mapping is surjective.

Proposition 4.7. $U: f \in \mathscr{H} \rightarrow\left[X_{f}\right] \in H_{\pi}^{1}\left(R^{2}\right)$ is surjective.

Proof. Let $X=a \frac{\partial}{\partial x}+b \frac{\partial}{\partial y},(a, b \in \mathscr{F})$ be any element of $Z_{\pi}^{1}\left(R^{2}\right)$. We must find an $f \in \mathscr{H}$ which satisfies

$$
x y \frac{\partial f}{\partial y}=-a, \quad x y \frac{\partial f}{\partial x}=b,
$$

where $a$ and $b$ satisfy (4.7). The integrability condition of $f$ is equivalent to (4.7). From this equation and the continuity of $a(x, y)$, we can see that $a(0, y)=0$ for any $y$. We can define a new $C^{\infty}$-function $\tilde{a}(x, y)$ by 


$$
\tilde{a}(x, y)= \begin{cases}\frac{a(x, y)}{x}, & (x \neq 0), \\ \frac{\partial a}{\partial x}(0, y), & (x=0) .\end{cases}
$$

Differentiating (4.7) with respect to $y$, and letting $y=0$, we have

$$
a(x, 0)=x \cdot \frac{\partial a}{\partial x}(x, 0) \text {. }
$$

Using this condition, we get, for $x \neq 0$,

$$
\begin{aligned}
\frac{d}{d x} \tilde{a}(x, 0) & =\lim _{h \rightarrow 0} \frac{\tilde{a}(x+h, 0)-\tilde{a}(x, 0)}{h} \\
& =\lim _{h \rightarrow 0} \frac{a(x+h, 0)-a(x, 0)}{h(x+h)}-\lim _{h \rightarrow 0} \frac{a(x, 0)}{x(x+h)} \\
& =\frac{1}{x^{2}}\left\{x \cdot \frac{\partial a}{\partial x}(x, 0)-a(x, 0)\right\} \\
& =0 .
\end{aligned}
$$

Hence by the continuity of $\tilde{a}(x, y)$, it follows that $\tilde{a}(x, 0)$ is constant for any $x$. Let $\tilde{a}(x, 0)=a_{1}$. Since $\tilde{a}(x, y)-a_{1}=0$ when $y=0$, there exists a $g(x, y) \in \mathscr{F}$ such that $\tilde{a}(x, y)$ can be written as

$$
\tilde{a}(x, y)=a_{1}+y \cdot g(x, y) .
$$

Thus for $x \neq 0$, we have $a(x, y) / x=a_{1}+y \cdot g(x, y)$. Recall that $a(0, y)=0$. Hence for any $x$, we finally obtain

$$
a(x, y)=a_{1} x+x y \cdot g(x, y) .
$$

By a similar argument, we also have

$$
b(x, y)=b_{1} y+x y \cdot h(x, y), \quad \exists h(x, y) \in \mathscr{F} .
$$

Note that $g(x, y)$ and $h(x, y)$ must satisfy

$$
\frac{\partial g}{\partial x}=-\frac{\partial h}{\partial y} .
$$

Then wa have 


$$
\begin{aligned}
d f & =\left(\frac{b_{1}}{x}+h\right) d x-\left(\frac{a_{1}}{y}+g\right) d y \\
& =d\left(b_{1} \log |x|-a_{1} \log |y|+l(x, y)\right),
\end{aligned}
$$

where $a_{1}$ and $b_{1}$ are constants, and $l(x, y) \in \mathscr{F}$ satisfies $\frac{\partial l}{\partial x}=h, \frac{\partial l}{\partial y}=-g$.

Let $\mathscr{F}_{c}$ be the space generated by $\mathscr{F}$ and constant functions on $R^{2} \backslash N$. Then we get

$$
f(x, y) \equiv b_{1} \log |x|-a_{1} \log |y|,\left(\bmod \mathscr{F}_{c}\right) .
$$

The funciton $f(x, y)$ is clearly an element of $\mathscr{H}$ and satisfies (4.9).

Theorem 4.8. If $\pi=\epsilon x y \frac{\partial}{\partial x} \wedge \frac{\partial}{\partial y}$, then $H_{\pi}^{1}\left(R^{2}\right) \cong R \oplus R$.

Proof. Since $U$ and $\iota^{*}$ are surjective by Proposition $4.7, H_{\pi}^{1}\left(R^{2}\right)$ is isomorphic to $\mathscr{H} / \mathscr{F}_{c}$. This space is spanned by $[f],\left(\bmod \mathscr{F}_{c}\right)$ for the function $f$ as defined by (4.15). More precisely, $H_{\pi}^{1}\left(R^{2}\right)$ is generated by the two vector fields $\left[x \frac{\partial}{\partial x}\right]$ and $\left[y \frac{\partial}{\partial y}\right]$.

\subsection{Case (4) $\left(R^{2}, \pi=y^{2} \frac{\partial}{\partial x} \wedge \frac{\partial}{\partial y}\right)$.}

It is also clear that $H_{\pi}^{0}\left(R^{2}\right) \cong R$. By the same method as in the case (3), we have $H_{\pi}^{1}\left(R^{2} \backslash(x\right.$-axis $\left.)\right)=0$. Thus the mapping $l^{*}: H_{\pi}^{1}\left(R^{2}\right) \rightarrow H_{\pi}^{1}\left(R^{2} \backslash\right.$ $(x$-axis $))=0$ is surjective, where $l: R^{2} \backslash(x$-axis $) \rightarrow R^{2}$ is the canonical inclusion. Let $X=a \frac{\partial}{\partial x}+b \frac{\partial}{\partial y}$ be an element of $Z_{\pi}^{1}\left(R^{2}\right)$. Then $a$ and $b$ satisfy

$$
2 b=y\left(\frac{\partial a}{\partial x}+\frac{\partial b}{\partial y}\right)
$$

A Hamiltonian vector field $\boldsymbol{X}_{\boldsymbol{f}}$ is given by

$$
X_{f}=y^{2}\left(-\frac{\partial f}{\partial y} \frac{\partial}{\partial x}+\frac{\partial f}{\partial x} \frac{\partial}{\partial y}\right)
$$

Next we define a space $\mathscr{K}$ by

$$
\mathscr{K}=\left\{f \in C^{\infty}\left(R^{2} \backslash(x-\text { axis })\right) \mid y^{2} \frac{\partial f}{\partial x}, y^{2} \frac{\partial f}{\partial y} \in \mathscr{F}\right\} .
$$

Then $\mathscr{K}$ contains $\mathscr{F}$ as its subspace. The linear mapping $V: f \in \mathscr{K} \rightarrow\left[\boldsymbol{X}_{f}\right]$ $\in H_{\pi}^{1}\left(R^{2}\right)$ is well-defined and $\operatorname{ker} V=\mathscr{F}_{c}$, where $\mathscr{F}_{c}$ is the space generated by 
and constant functions on $R^{2} \backslash(x$-axis $)$.

Theorem 4.9. If $\pi=y^{2} \frac{\partial}{\partial x} \wedge \frac{\partial}{\partial y}$, then $H_{\pi}^{1}\left(R^{2}\right) \cong \mathscr{K} / \mathscr{F}_{c}$, and is of infinite dimension.

Proof. First we prove that $V$ is surjective. Let $X=a \frac{\partial}{\partial x}+b \frac{\partial}{\partial y}$ be any element of $Z_{\pi}^{1}\left(R^{2}\right)$. Then we must find a solution $f \in \mathscr{K}$ such that

$$
y^{2} \frac{\partial f}{\partial y}=-a, \quad y^{2} \frac{\partial f}{\partial x}=b .
$$

The integrability condition of $f$ is equivalent to (4.16). Let $\int \frac{\partial a}{\partial x} d x=a+\theta(y)$. Then the desired function $f$ is given by

$$
f(x, y)=\frac{1}{y^{2}} \int b(x, y) d x+\int \frac{\theta(y)}{y^{2}} d y .
$$

In fact, by using (4.16), we know that the function $f$ is an element of $\mathscr{K}$, and that $f$ satisfies (4.18). Thus the linear mapping $V$ is surjective. Now it is clear that $H_{\pi}^{1}\left(R^{2}\right)$ is isomorphic to $\mathscr{K} / \mathscr{F}_{c}$. Let $m(x, y)$ be any function of $\mathscr{F}$ such that $m(x, 0) \neq 0$. Then $m(x, y) / y$ is contained in $\mathscr{K}$, but it is not contained in $\mathscr{F}_{c}$. Hence $\mathscr{K} / \mathscr{F}_{c}$ is of infinite dimension.

\section{§5. Computations of $H_{\pi}^{2}\left(R^{2}\right)$}

First note that any two-vector field $f(x, y) \frac{\partial}{\partial x} \wedge \frac{\partial}{\partial y}$ must be a cocycle. Thus $Z_{\pi}^{2}\left(R^{2}\right)$, the space of 2-cocycles, is isomorphic to $\mathscr{F}=C^{\infty}\left(R^{2}\right)$. In the previous section, we determined $H_{\pi}^{2}\left(R^{2}\right)$ for case (1). So let's start with case (2).

Throughout this section, we denote the space of $C^{\infty}$-functions which are flat at the origin by $\mathscr{I}$.

5.1. Case (2) $\left(R^{2}, \pi=\delta\left(x^{2}+y^{2}\right) \frac{\partial}{\partial x} \wedge \frac{\partial}{\partial y}\right)$.

We define a subspace $\mathscr{F}^{\prime}$ of $\mathscr{F}$ as follows:

$$
\mathscr{F}^{\prime}=\left\{\left(x^{2}+y^{2}\right)\left(\frac{\partial a}{\partial x}+\frac{\partial b}{\partial y}\right)-2(x a+y b) \mid a, b \in \mathscr{F}\right\} .
$$

Let $X=a \frac{\partial}{\partial x}+b \frac{\partial}{\partial y}$ be an element of $\chi\left(R^{2}\right)$. Since $D(X)=[\pi, X]=\delta\left\{\left(x^{2}+y^{2}\right)\left(\frac{\partial a}{\partial x}+\frac{\partial b}{\partial y}\right)\right.$ $-2(x a+y b)\} \frac{\partial}{\partial x} \wedge \frac{\partial}{\partial y}$, the space of 2-coboundaries, $B_{\pi}^{2}\left(R^{2}\right)$, is isomorphic to $\mathscr{F}^{\prime}$. Thus we have $H_{\pi}^{2}\left(R^{2}\right) \cong \mathscr{F} / \mathscr{F}^{\prime}$. For the sake of simplicity of description, we define two differential operators, $D_{1}$ and $D_{2}$, by 


$$
\begin{aligned}
& D_{1} a=\left(x^{2}+y^{2}\right) \frac{\partial a}{\partial x}-2 x a, \\
& D_{2} a=\left(x^{2}+y^{2}\right) \frac{\partial a}{\partial y}-2 y a .
\end{aligned}
$$

Then $q \in \mathscr{F}$ is contained in $\mathscr{F}^{\prime}$ if and only if there exist $a, b \in \mathscr{F}$ such that:

$$
D_{1} a+D_{2} b=q \text {. }
$$

Lemma 5.1. $\mathscr{I}$ is a subspace of $\mathscr{F}^{\prime}$.

Proof. Let $q \in \mathscr{I}$. Then $\frac{q}{\left(x^{2}+y^{2}\right)^{2}}$ is contained in $\mathscr{F}$. For example, let $a=\left(x^{2}+y^{2}\right) \int \frac{q}{\left(x^{2}+y^{2}\right)^{2}} d x$, and $b=0$. Then $a$ and $b$ satisfy (5.1). Thus $q$ is contained in $\mathscr{F}^{\prime}$.

Let $F$ be the ring of formal power series generated by formal Taylor expansions of all elements of $\mathscr{F}$ at the origin. Define a subspace $F^{\prime}$ of $F$ by

$$
F^{\prime}=\left\{D_{1} A+D_{2} B \mid A, B \in F\right\} \text {. }
$$

Then we have

Proposition 5.2. $F^{\prime}$ is of codimension 2 in $F$.

Proof. Our aim is to consider whether the following equation:

$$
D_{1} A+D_{2} B=Q
$$

can be solved or not for every homogeneous term of $Q$. Let $A$ and $B$ be polynomials of degree 1 . Then it is clear that the space $\left\{D_{1} A+D_{2} B\right\}$ spans a 4-dimensional space $\left\langle x, y, x^{2}-y^{2}, x y\right\rangle$. (To be specific, $D_{1} A+D_{2} B$ can not attain to $x^{2}+y^{2}$.) Next let $Q$ be any homogeneous polynomial of degree $n$, $(n \geq 3)$. Now we will show that the equation (5.2) can be solved (i.e., we can find homogeneous polynomials $A$ and $B$ which satisfy (5.2)). Let

$$
\begin{aligned}
& A=\sum_{t+s=n-1} A_{t, s} x^{t} y^{s}, \\
& B=\sum_{t+s=n-1} B_{t, s} x^{t} y^{s},
\end{aligned}
$$




$$
Q=\sum_{k+l=n} Q_{k, l} x^{k} y^{l}
$$

Substituting $A, B$ and $Q$ into (5.2), we obtain a system of $(n+1)$-linear equations. With respect to the basis $\left\langle y^{n}, x y^{n-1}, \cdots, x^{n-1} y, x^{n}\right\rangle$, the matrix of coefficients is as follows:

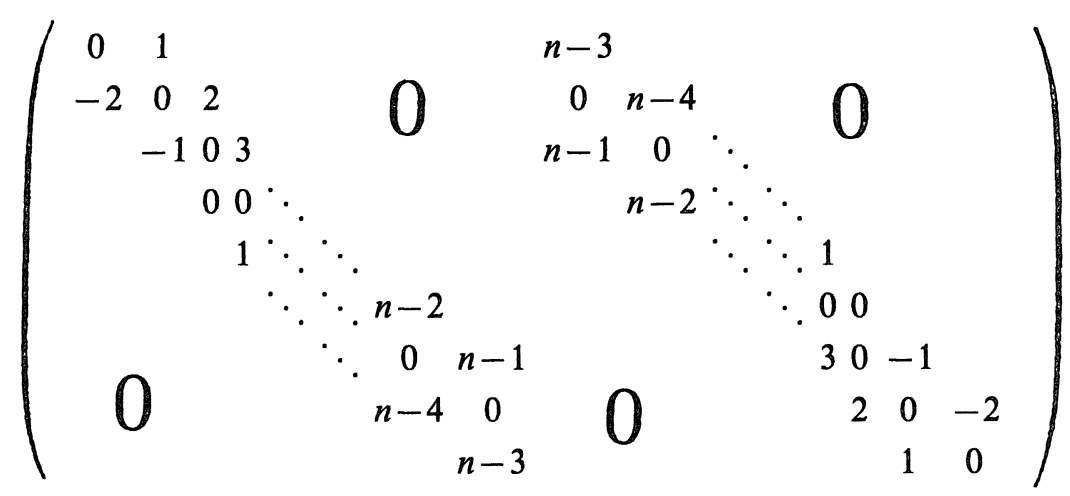

The above matrix, of type $(n+1) \times 2 n$, which we denote by $C(n+1,2 n)$, has rank $n+1$. Hence we can find $A$ and $B$ satisfying (5.2). Thus for any $Q \in F$ with $\operatorname{deg} Q \geq 3$, we can solve (5.2). It can be concluded that $F / F^{\prime} \cong\left\langle 1, x^{2}+y^{2}\right\rangle$.

Let $E: \mathscr{F} \rightarrow F$ be the linear map defined by $f(x, y) \in \mathscr{F} \mapsto[f(x, y)]_{(0,0)}$. Then by one of the theorems of E. Borel, $E$ is surjective.

Lemma 5.3. In Equation (5.1), let $E(q)=Q$. If the formal equation (5.2) has a solution, then (5.1) also has a solution.

Proof. Choose $\bar{a}, \bar{b} \in \mathscr{F}$ such that $E(\bar{a})=A$ and $E(\bar{b})=B$. Then if we let $D_{1} \bar{a}+D_{2} \bar{b}-q=\tilde{q}, \tilde{q}$ is an element of $\mathscr{I}$. Thus by Lemma 5.1 , there exists $\tilde{a} \in \mathscr{F}$ such that $D_{1} \tilde{a}=\tilde{q}$. Hence we have $D_{1}(\bar{a}-\tilde{a})+D_{2} \bar{b}=q$.

Corollary 5.4. $E\left(\mathscr{F}^{\prime}\right)=F^{\prime}$.

Lemma 5.5. $E^{-1}\left(F^{\prime}\right)=\mathscr{F}^{\prime}$.

Proof. By Corollary 5.4, we obtain $E^{-1}\left(F^{\prime}\right) \supset \mathscr{F}^{\prime}$. Hence, it suffices to 
prove that $E^{-1}\left(F^{\prime}\right) \subset \mathscr{F}^{\prime}$. Let $q_{1}$ be any element of $E^{-1}\left(F^{\prime}\right)$ and let $E\left(q_{1}\right)=Q_{1}$. Then, by Corollary 5.4, there exists $q_{2} \in \mathscr{F}^{\prime}$ such that $E\left(q_{2}\right)=Q_{1}$. Thus $q_{1}-q_{2} \in \mathscr{I}$. Using Lemma 5.1, we obtain that $q_{1} \in \mathscr{F}^{\prime}$.

Theorem 5.6. Let $\pi=\delta\left(x^{2}+y^{2}\right) \frac{\partial}{\partial x} \wedge \frac{\partial}{\partial y}$. Then $H_{\pi}^{2}\left(R^{2}\right) \cong R \oplus R$.

Proof. Combining Proposition 5.2 and Lemma 5.5, we can see that

$$
H_{\pi}^{2}\left(R^{2}\right) \cong \mathscr{F} / \mathscr{F}^{\prime} \cong \mathscr{F} / E^{-1}\left(F^{\prime}\right) \cong F / F^{\prime} \cong\left\langle 1, x^{2}+y^{2}\right\rangle \cong R \oplus R .
$$

5.2. Case (3) $\left(R^{2}, \pi=\epsilon x y \frac{\partial}{\partial x} \wedge \frac{\partial}{\partial y}\right)$. Define a subspace $\mathscr{F}^{\prime \prime}$ of $\mathscr{F}$ by

$$
\mathscr{F}^{\prime \prime}=\left\{x y\left(\frac{\partial a}{\partial x}+\frac{\partial b}{\partial y}\right)-(y a+x b) \mid a, b \in \mathscr{F}\right\} .
$$

Note that since $D(X)=\epsilon\left\{x y\left(\frac{\partial a}{\partial x}+\frac{\partial b}{\partial y}\right)-(y a+x b)\right\} \frac{\partial}{\partial x} \wedge \frac{\partial}{\partial y}$ for $X=a \frac{\partial}{\partial x}+b \frac{\partial}{\partial y}$, the space of 2-coboundaries $B_{\pi}^{2}\left(R^{2}\right)$ is isomorphic to $\mathscr{F}^{\prime \prime}$. Accordingly $H_{\pi}^{2}\left(R^{2}\right)$ is isomorphic to $\mathscr{F} / \mathscr{F}^{\prime \prime}$. Consider the following differential equation:

$$
x y\left(\frac{\partial a}{\partial x}+\frac{\partial b}{\partial y}\right)-(y a+x b)=q,
$$

where $a, b, q \in \mathscr{F}$.

First we show that (5.3) has a solution if $q$ is flat at the origin.

Lemma 5.7. I is a subspace of $\mathscr{F}^{\prime \prime}$.

Proof. For any $f \in \mathscr{F}$, let $a_{1}=\int f d x$. Substituting $a=x a_{1}$ and $b=0$ into (5.3), we have $x^{2} y f \in \mathscr{F}^{\prime \prime}$. On the other hand, let $b_{1}=\int f d y$. Substituting $a=0$ and $b=y b_{1}$ into (5.3) yields $x y^{2} f \in \mathscr{F}^{\prime \prime}$. Thus we obtain

$$
x^{2} y f \in \mathscr{F}^{\prime \prime}, \quad x y^{2} f \in \mathscr{F}^{\prime \prime} .
$$

Next let $a=0$ and $b=x f$. Combining (5.3) and (5.4), we get $x^{2} \mathscr{F} \subset \mathscr{F} "$ and $y^{2} \mathscr{F} \subset \mathscr{F}^{\prime \prime}$. Thus we obtain

$$
x^{2} \mathscr{F} \subset \mathscr{F}^{\prime \prime}, \quad y^{2} \mathscr{F} \subset \mathscr{F}^{\prime \prime} .
$$

Let $f$ be any element of $\mathscr{I}$. Since $\frac{f}{x^{2}+y^{2}}$ is also an element of $\mathscr{I}$, it follows from Equation (5.5) that $f=\left(x^{2}+y^{2}\right) \frac{f}{x^{2}+y^{2}} \in \mathscr{F}^{\prime \prime}$. 
As in the previous case, we define a subspace $F^{\prime \prime}$ of $F$ by

$$
F^{\prime \prime}=\left\{x y\left(\frac{\partial A}{\partial x}+\frac{\partial B}{\partial y}\right)-(y A+x B) \mid A, B \in F\right\} .
$$

Proposition 5.8. $F^{\prime \prime}$ is of codimension 2 in $F$.

Proof. First note that $F^{\prime \prime}$ contains all linear functions, but it does not contain $x y$ nor constants. By the same method as in the proof of Proposition 5.2 , we have a matrix $D(n+1,2 n)$ of type $(n+1) \times 2 n$ :

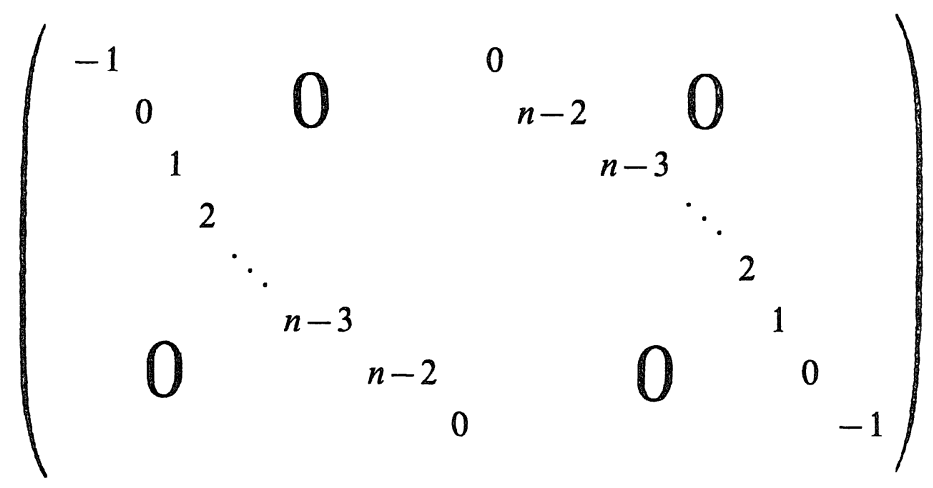

Since rank $D(n+1,2 n)=n+1$, it follows that $F^{\prime \prime}$ contains all formal functions $Q$ of $\operatorname{deg} Q \geq 3$. And finally we get $F / F^{\prime \prime} \cong\langle 1, x y\rangle$.

Theorem 5.9. Let $\pi=\epsilon x y \frac{\partial}{\partial x} \wedge \frac{\partial}{\partial y}$. Then $H_{\pi}^{2}\left(R^{2}\right) \cong R \oplus R$.

Proof. It is clear that $E\left(\mathscr{F}^{\prime \prime}\right)=F^{\prime \prime}$. From Lemma 5.7, it follows that $E^{-1}\left(F^{\prime \prime}\right)=\mathscr{F}$. Combining these facts with Proposition 5.8, we get

$$
H_{\pi}^{2}\left(R^{2}\right) \cong \mathscr{F} / \mathscr{F} " \cong \mathscr{F} / E^{-1}\left(F^{\prime \prime}\right) \cong F / F^{\prime \prime} \cong\langle 1, x y\rangle \cong R \oplus R .
$$

5.3. Case (4) $\left(R^{2}, \pi=y^{2} \frac{\partial}{\partial x} \wedge \frac{\partial}{\partial y}\right)$. In this case, a subspace $\mathscr{F}^{\prime \prime \prime}$ of $\mathscr{F}$ is defined by

$$
\mathscr{F}^{\prime \prime \prime}=\left\{y^{2}\left(\frac{\partial a}{\partial x}+\frac{\partial b}{\partial y}\right)-2 y b \mid a, b \in \mathscr{F}\right\} .
$$

Since $D(X)=\left\{y^{2}\left(\frac{\partial a}{\partial x}+\frac{\partial b}{\partial y}\right)-2 y b\right\} \frac{\partial}{\partial x} \wedge \frac{\partial}{\partial y}$ for $X=a \frac{\partial}{\partial x}+b \frac{\partial}{\partial y}$, the space of all 2coboundaries, or the space $B_{\pi}^{2}\left(R^{2}\right)$, is isomorphic to $\mathscr{F}^{\prime \prime \prime}$, and $H_{\pi}^{2}\left(R^{2}\right)$ is 
isomorphic to $\mathscr{F} / \mathscr{F}^{\prime \prime \prime}$.

Theorem 5.10. Let $\mathscr{F}(x)$ be the space of $C^{\infty}$-functions of one variable x. Then $H_{\pi}^{2}\left(R^{2}\right) \cong \mathscr{F}(x)$.

Proof. For any function $f \in \mathscr{F}$, let $b=-\frac{1}{2} f$ and $a=-\int \frac{\partial b}{\partial y} d x$. Then $y^{2}\left(\frac{\partial a}{\partial x}+\frac{\partial b}{\partial y}\right)-2 y b=y f \in \mathscr{F}^{\prime \prime \prime}$. Hence $y \mathscr{F} \subset \mathscr{F}^{\prime \prime \prime}$. The converse of this last is also clear. Thus we know that $\mathscr{F}^{\prime \prime \prime}=y \mathscr{F}$. Let us define a linear mapping $\mathscr{L}: \mathscr{F} \rightarrow \mathscr{F}(x)$ by $f(x, y) \mapsto f(x, 0)$. Then $\mathscr{L}$ is surjective. It is easy to see that $\operatorname{ker} \mathscr{L}=y \mathscr{F}=\mathscr{F}^{\prime \prime \prime}$. Thus we obtain that $H_{\pi}^{2}\left(R^{2}\right) \cong \mathscr{F} / \mathscr{F}^{\prime \prime \prime} \cong \mathscr{F}(x)$.

Remark. For a Poisson manifold $(M, \pi), H_{\pi}^{2}(M)$ has the distinguishing element $[\pi]$. If $[\pi]=0,(M, \pi)$ is called a homogeneous Poisson manifold (or an exact Poisson manifold). Through the considerations above, we know that:

a) If $\pi=0$ or $\pi=y^{2} \frac{\partial}{\partial x} \wedge \frac{\partial}{\partial y}$, then $\left(R^{2}, \pi\right)$ is homogeneous,

b) If $\pi=\delta\left(x^{2}+y^{2}\right) \frac{\partial}{\partial x} \wedge \frac{\partial}{\partial y}$ or $\pi=\epsilon x y \frac{\partial}{\partial x} \wedge \frac{\partial}{\partial y}$, then $\left(R^{2}, \pi\right)$ is not homogeneous.

\section{References}

[ 1 ] Bhaskara, K. H. and Rama, K., Quadratic Poisson structures, J. Math. Phys., 32 (1991), 2319-2322.

[ 2 ] Dufour, J.P. et Haraki, A., Rotationnels et structures de Poisson quadratiques, $C . R$. Acad. Sci Paris Série I Math., 312 (1991), 137-140.

[ 3 ] Ginzburg, V.L. and Weinstein, A., Lie-Poisson structure on some Poisson Lie groups, $J$. Amer Math. Soc., 5 (1992), 445-453.

[ 4 ] Ginzburg, V.L., Momentum mappings and Poisson cohomology, Preprint (1995).

[ 5 ] Karasev, M.V., Analogues of objects of the Lie group theory for nonlinear Poisson brackets, Soviet Math. Izvestia, 28 (1987), 497-527.

[6] Lichnerowicz, A., Les variétés de Poisson et leurs algèbres de Lie associées, J. Differential Geom., 12 (1977), 253-300.

[ 7 ] Liu, Z-J. and Xu, P., On quadratic Poisson structures, Lett. in Math. Phys., 26 (1992), 33-42.

[8] Lu, J.H., Multiplicative and affine Poisson structures on Lie groups, Thesis, Univ. of California, Berkeley, (1990).

[9] Nakanishi, N., On the structure of infinitesimal automorphisms of linear Poisson manifolds I, J. Math. Kyoto Univ., 31 (1991), 71-82.

[10] , Poisson cohomology, (in Japanese), RIMS Kökyüroku, 875 (1994), 156-167.

[11] Integrability of infinitesimal automorphisms of linear Poisson manifolds, Proc. Japan Acad. Ser. A Math. Sci., 71, (1995), 119-122.

[12] Vaisman, I., Remarks on the Lichnerowicz-Poisson cohomology, Ann. Inst. Fourier Grenoble, 40 (1990), 951-963.

[13] - Lectures on the geometry of Poisson manifolds, Birkhauser, 1994.

[14] Xu, P., Poisson cohomology of regular Poisson manifolds, Ann. Inst. Fourier Grenoble, 42 (1992), 967-988. 
